Häußler, Angela

\title{
Lässt sich Gesundheit lehren?
}

Haushalt in Bildung \& Forschung 4 (2015) 2, S. 31-42

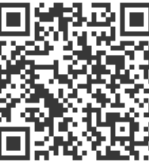

Quellenangabe/ Reference:

Häußler, Angela: Lässt sich Gesundheit lehren? - In: Haushalt in Bildung \& Forschung 4 (2015) 2, S. 31-42 - URN: urn:nbn:de:0111-pedocs-203704 - DOI: 10.25656/01:20370

https://nbn-resolving.org/urn:nbn:de:0111-pedocs-203704

https://doi.org/10.25656/01:20370

in Kooperation mit / in cooperation with:

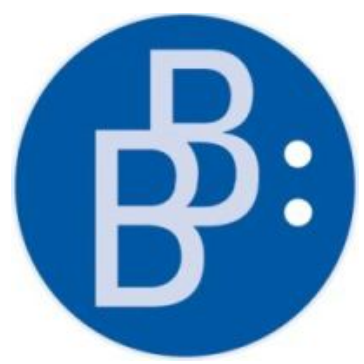

https://www.budrich.de

\section{Nutzungsbedingungen}

Gewährt wird ein nicht exklusives, nicht übertragbares, persönliches und beschränktes Recht auf Nutzung dieses Dokuments. Dieses Dokument ist ausschließlich für den persönlichen, nicht-kommerziellen Gebrauch bestimmt. Die Nutzung stellt keine Übertragung des Eigentumsrechts an diesem Dokument dar und gilt vorbehaltlich der folgenden Einschränkungen: Auf sämtlichen Kopien dieses Dokuments müssen alle Urheberrechtshinweise und sonstigen Hinweise auf gesetzlichen Schutz beibehalten werden. Sie dürfen dieses Dokument nicht in irgendeiner Weise abändern, noch dürfen Sie dieses Dokument für öffentliche oder kommerzielle Zwecke vervielfältigen, öffentlich ausstellen, aufführen, vertreiben oder anderweitig nutzen.

Mit der Verwendung dieses Dokuments erkennen Sie die Nutzungsbedingungen an.

\section{Terms of use}

We grant a non-exclusive, non-transferable, individual and limited right to using this document.

This document is solely intended for your personal, non-commercial use. Use of this document does not include any transfer of property rights and it is conditional to the following limitations: All of the copies of this documents must retain all copyright information and other information regarding legal protection. You are not allowed to alter this document in any way, to copy it for public or commercial purposes, to exhibit the document in public, to perform, distribute or otherwise use the document in public.

By using this particular document, you accept the above-stated conditions of use.

\section{Kontakt / Contact:}

\section{peDOcs}

DIPF | Leibniz-Institut für Bildungsforschung und Bildungsinformation Informationszentrum (IZ) Bildung

E-Mail:pedocs@dipf.de

Internet: www.pedocs.de

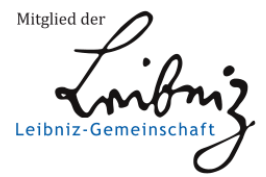


4. Jahrgang Heft 2 2015

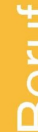

m

(1)

$\frac{\sqrt{2}}{\frac{2}{c}}$

$>$

(1)

(1)

ते

(1)

है

(1)

$\frac{1}{2}$

3

$>$

(1)

$\frac{8}{8}$

$\frac{1}{c}$

है

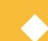

필

$\checkmark$

$\frac{\overline{1}}{\frac{1}{0}}$

है

(1)

(1)
है

$\frac{d}{\frac{c}{c}}$

\section{Haushalt in}

Forschung

Wa(h)re Gesundheit
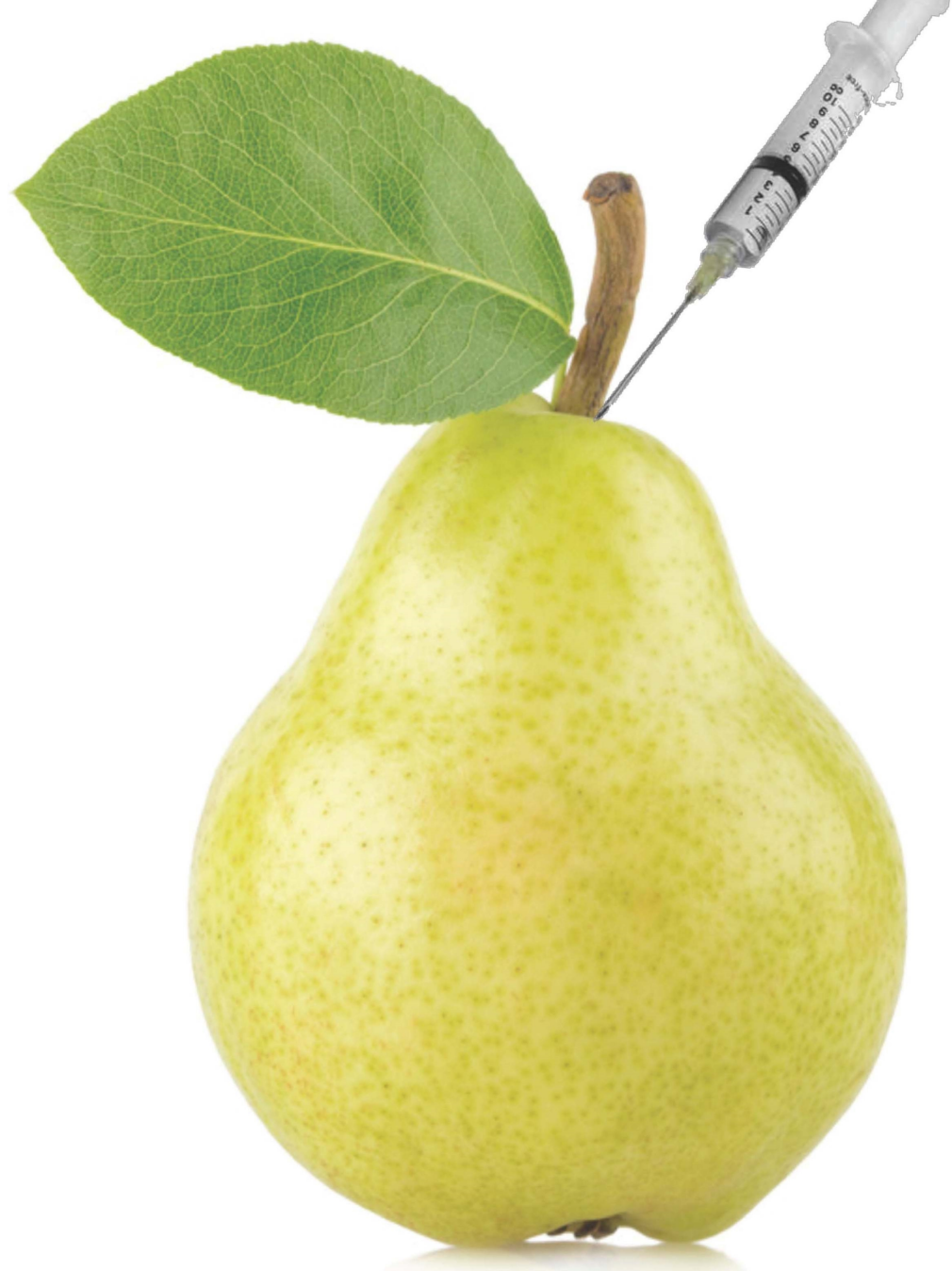
Inhaltsverzeichnis |

Gabriela Leitner

Editorial...... 2

Gabriela Leitner \& Maria Schuh

Ernährungs- und Verbraucherbildung - quo vadis? 3

Kirsten Schlegel-Matthies

Gesundheit und Selbstverantwortung - Was kann und was sollte gelehrt werden?. 18

Angela Häußler

Lässt sich Gesundheit lehren?.

Ute Bender

„Eating Smart“ - Funktionen von Ernährung in der (schulischen)

Gemeinschaftsverpflegung.

Ursula Buchner

Lernwege in der Schulküche. 58

Workshopnachlese 73

Theres Rathmanner

Ernährungsprojekte in Schulen weltweit - ein Blick über den Tellerrand. 86

Michaela Kropatschek

Spielregeln für Werbung im Lebensmittelbereich und in der Schule.

Maya Lucia Joray, Michèle Simone Leuenberger \& Zeno Stanga

Ursachen und Strategien zur Bekämpfung von Übergewicht und Adipositas

bei Kindern und Jugendlichen - aus der Sicht der Schule.

Silke Bartsch \& Werner Brandl

Von der Didaktischen Rekonstruktion zu einer

Didaktik subjektorientierten Lernens und Lehrens.

Rezensionen

Thomas Mohrs

Nachhaltige Lebensstile.... 126

Regine Bigga

Was der Mensch essen darf...... 


\section{Angela Häußler}

\section{Lässt sich Gesundheit lehren?}

Zunächst wird die Ausgangssituation der EVB im Umgang mit der Subjektivität lebensweltlicher Themen an Schulen und Hochschulen aus der Perspektive aller Beteiligten dargestellt und besondere hochschuldidaktische Herausforderungen skizziert. Im zweiten Teil des Beitrags wird exemplarisch am Beispiel der Normativität des Gesundheitsbegriffs eine Standortbestimmung für die Lehramtsausbildung als Diskursbeitrag formuliert.

Schlüsselwörter: Lehrerinnen- und Lehrerbildung, subjektive Theorien, Gesundheitsnormen, Umgang mit Heterogenität

\section{Ernährungs- und Verbraucherbildung: spannend und spannungsgeladen}

In der schulischen Ernährungs- und Verbraucherbildung (EVB) können Lehrerinnen und Lehrer für die Planung und Gestaltung des Unterrichts einen großen Vorteil nutzen: Der Lebensweltbezug ist bei alltagskulturellen Themen in der Regel direkt gegeben und muss nicht erst didaktisch konstruiert werden. Essen, Kleidung und Konsum sind alltägliche Erfahrungsfelder für Kinder und Jugendliche und bieten vielfältige Möglichkeiten, um im Unterricht daran anzuknüpfen.

Gleichzeitig ist mit dem Lebensweltbezug jedoch eine besondere Problematik verbunden: Die Vorerfahrungen der Schülerinnen und Schüler können sehr unterschiedlich sein, der Zugang zu den Handlungsfeldern ist durch subjektive Überzeugungen und die alltäglich erlebte/gelebte Praxis durch kaum reflektierte Gewohnheiten und Routinen geprägt. ${ }^{1}$ Diese Ausgangslage macht die EVB zu einem gleichermaßen spannenden wie spannungsgeladenen Fach mit besonderen didaktischen Herausforderungen für Lehrkräfte und damit auch für die Lehramtsausbildung an den Hochschulen. Angehende Lehrerinnen und Lehrer benötigen z.B. Kompetenzen für den Umgang mit Heterogenität, ein tiefes Verständnis für die Komplexität des Alltagshandelns sowie reflexive Kompetenzen zur Gewordenheit und Geschichte der alltäglichen Verhaltensweisen, z.B. beim Essen. Fachdidaktischen Konzepte der Ernährungs- und Verbraucherbildung setzen an dieser Stelle an. REVIS formuliert die reflektierte Auseinandersetzung mit der eigenen Essbiografie als zentrales Bildungsziel und das Konzept der Subjektorientierung stellt den Lebensweltbezug im EVB-Unterricht aus der Perspektive von Schülerinnen und 


\section{Lässt sich Gesundheit lehren?}

Schülern ins Zentrum (Bartsch, 2012; Fachgruppe Ernährungs- und Verbraucherbildung 2005).

Aufgrund der sehr begrenzten Zeit, die für die Ausbildung angehender Lehrkräfte an der Hochschule zur Verfügung steht, stellt sich jedoch die Frage, wie dieser didaktischen Herausforderung begegnet werden kann. Im vorliegenden Beitrag möchte ich eine Standortbestimmung der hochschuldidaktischen Herausforderungen in der EVB als Diskursbeitrag versuchen und dabei besonders den Aspekt der Normativität von Gesundheitskonzepten in den Blick nehmen.

\subsection{Die Perspektive der Schülerinnen und Schüler}

Wenn in der Schule Ernährungsthemen auf dem Stundenplan stehen, steht damit auch die „gesunde Ernährung“ mit im Klassenraum. Schülerinnen und Schüler erwarten in dieser Situation die Vermittlung von Wissen über gesunde, gute, richtige Ernährung. Die grundlegenden Regeln sind dabei schon Kindern im Grundschulalter bekannt. Diese Erwartungshaltung ist nicht überraschend, da Wissensvermittlung im schulischen Kontext nach wie vor einen großen Raum einnimmt und die jeweiligen Bildungsinhalte auf Lerninhalte heruntergebrochen werden. Die auch von den Schülerinnen und Schülern verinnerlichte disziplinäre Struktur von Lerninhalten führt weiterhin dazu, dass eine multiperspektivische Betrachtung, die für eine umfassende Annäherung an alltagskulturelle Themen als Grundlage für Bildungsprozesse notwendig ist, nicht direkt in die strukturelle Logik der Schule einzupassen ist.

\subsection{Perspektive der Studierenden}

Die meisten Studierenden in den Lehramtsberufen der EVB sind sich ihrerseits schon früh der Brisanz des abstrakten Gesundheitsbegriffs in der Ernährungsbildung bewusst. Die Erfahrung aus Lehrveranstaltungen zeigt, dass Studierende schon zu Beginn des Studiums ahnen, dass das Konzept der „gesunden Ernährung“ in Form von Empfehlungen wenig Anschluss an die alltäglichen Lebenswelten von Kindern und Jugendlichen hat und sie verstehen in der Regel die sehr begrenzte Handlungsrelevanz von Wissen in diesem Bereich.

Gleichzeitig ist für viele Studierende der Wunsch, Kompetenzen für eine gesunde Lebensführung an Kinder und Jugendliche vermitteln zu können, eine wichtige Motivation für die Wahl des Studienfachs. In einer Umfrage ${ }^{2}$ unter Erstsemestern des Studienfachs „Alltagskultur und Gesundheit“ an der Pädagogischen Hochschule Heidelberg haben Studierende Wahl des Faches zum Beispiel mit folgenden Aussagen begründet: 


\section{Lässt sich Gesundheit lehren? |}

„Das Wissen über die Eigenschaften verschiedener Nahrungsmittel und die Möglichkeit, sich bewusst gesund ernähren zu können, sind Dinge, die den Kindern in der Schule unbedingt vermittelt werden sollen.“

„Aspekte des Fachs zielen genau auf aktuelle gesellschaftliche Probleme (falsche Ernährung, fehlende Verbraucherbildung).“

„Außerdem erachte ich dieses Fach als besondere Möglichkeit, junge Menschen für einen bewussten Umgang mit dem eigenen Leben sowie der Umwelt zu sensibilisieren. Dies sollte im Zentrum der Erziehung von Kindern und Jugendlichen stehen und ist in keinem anderen Schulfach so praktisch und lebensnah zu gestalten wie in diesem!“

Besonders in dem letzten Zitat drückt sich die eingangs beschriebene Ambivalenz der EVB noch einmal konkret aus. Die Studierenden erkennen die besonderen Möglichkeiten des lebensweltlichen Zugangs, gleichzeitig wird auch die besondere Problematik deutlich. So verwenden die Studierenden subjektiv geprägte, normative Begriffe wie „falsche Ernährung“ oder den ,gesunden, bewussten Umgang mit Ernährung und der Umwelt"،3.

\subsection{Perspektive der Lehrerinnen- und Lehrerbildung}

Für hochschuldidaktische Ansätze ist die hohe Motivation der Studierenden für die lebenspraktische Ernährungs- und Verbraucherbildung eine gut nutzbare Ausgangssituation. Gleichzeitig ist jedoch der Zugang zu den Inhalten nicht nur bei den Schülerinnen und Schülern, sondern auch bei den Studierenden durch subjektive Alltagsvorstellungen und normative Wertorientierungen bestimmt. Diese wurden biografisch erworben und sind stark durch die soziale Herkunft mit bestimmt und erweisen sich als recht robust (Bender \& Hertrampf, 2014, S. 6). Weiterhin werden die subjektiven Überzeugungen auch durch kollektive, medial vermittelte Vorstellungen, zum Beispiel zur gesunden Ernährung geprägt.

Dies erleben Studierende nicht selten auch in ihren schulpraktischen Erfahrungen so. Suter und Högger beschreiben, dass Lehrkräfte in der Ernährungsbildung in konkreten Unterrichtssituationen oft nicht auf ein systematisch entwickeltes und reflektiertes Konzept zurück greifen können, sondern Alltagsvorstellungen nutzen, die Teil des kollektiven Gedankenguts über die Übergewichtsproblematik und gesundes Ernährungsverhalten sind (Suter \& Högger, 2014, S. 17). Damit ist eine grundlegende Voraussetzung für eine gelingende EVB nicht gegeben, da unreflektiert transportierte subjektive Alltagsvorstellungen und Wertorientierungen der Lehrkräfte in der Regel nicht anschlussfähig sind an die Heterogenität der alltäglichen Lebenswelt von Schülerinnen und Schülern (Bender \& Hertrampf, 2014, S. 12f.). Eine umfassende Reflektion und ein tiefes Verständnis des jeweils subjektiven und normativen Charakters lebensweltlicher Themen sowie der Komplexität der Bedingungen und Strukturen alltäglichen Handelns stellt eine zentrale Voraus- 


\section{Lässt sich Gesundheit lehren?}

setzung für eine erfolgreiche EVB dar und ist ein wichtiger Aspekt der professionellen Kompetenz von Lehrkräften (ebd., S. 6).

Im folgenden Abschnitt sollen die komplexen Dimensionen der Subjektivität und Normativität beispielhaft am Gesundheitsbegriff gezeigt werden

\section{Subjektivität und Normativität in der EVB am Beispiel des Gesundheitsbegriffs}

\subsection{Der Paradigmenwechsel von der Erziehung zur Bildung}

In den Feldern der Ernährungs-, Verbraucher- oder auch der Gesundheitsbildung hat sich in den vergangenen Jahrzehnten ein Paradigmenwechsel vollzogen. So wurden die explizit normgeleiteten und auf Verhaltensänderungen im Sinne des „richtigen“ Verhaltens ausgelegten Erziehungskonzepte durch umfassende Bildungskonzepte abgelöst (Schlegel-Matthies, 2004, S. 7f.).

„Erziehung ist eine beabsichtigte, normgeleitete und gelenkte Vermittlung von Wissen und Verhaltensregeln, z.B. in der Familie, der Schule, im Beruf, in der Freizeit

Bildung ist die Auseinandersetzung des Menschen mit sich selbst und seiner kulturellen, materiell-dinglichen, sozialen Umwelt Sie findet nicht nur in bildungsrelevanten Institutionen, sondern auch im Alltag statt." (Bartsch, Büning-Fesel \& Cremer et al., 2013, S. M85)

Diese Entwicklung der EVB steht damit nicht außerhalb des allgemeinen Bildungsverständnisses, auch hier hat sich ein umfassender Bildungsbegriff im Sinne der oben genannten Definition durchgesetzt ${ }^{4}$. (Klafki, 2007; Tenorth \& Tippelt, 2007, S. 94) Wie schon im ersten Teil des Beitrags gezeigt, sind die Themen der EVB durch den Lebensweltbezug und die Komplexität der Inhalte bei allen Bildungsakteuren durch biografisch erworbene, subjektive und normative Einstellungen und Haltungen geprägt. Normativität ist in der Entwicklung vom Erziehungs- zum Bildungsbegriff also nicht verschwunden, kommt in der Bildungsdefinition nicht explizit zur Sprache. Bildung beinhaltet weiterhin immer auch Erziehung im Sinne einer gelenkten Beeinflussung. Wie hat sich der Umgang mit Normen den komplexen Themenfeldern Ernährung, Gesundheit und Konsum im Zuge dieses Paradigmenwechsels verändert und was bedeutet das für die EVB? Haben emanzipatorische und kompetenzorientierte Bildungskonzepte dazu geführt, dass mit der Abkehr von festgelegten normativen Erziehungszielen normativ geprägte Haltungen und Wertorientierung unsichtbar geworden sind? Und welche kollektiven Überzeugungen fließen in die Entwicklung von subjektiven Überzeugungen und normativen Haltungen mit ein? Diese Fragen sind erkenntnisleitend bei der folgenden Auseinandersetzung mit dem Gesundheitsbegriff. Dabei sollen besonders zwei Diskurslinien verfolgt werden, die 


\section{Lässt sich Gesundheit lehren?}

im Zusammenhang mit dem übergeordneten Thema „Ethik und Gesundheit“ besonders interessant sind. Als Thesen lassen sie sich so formulieren:

- Das jeweilige Verständnis von Gesundheit und gesunder Lebensführung ist ein soziales Konstrukt, welches auf normativen Setzungen beruht

- Gesundheits-, Ernährungs- und Verbraucherbildung bedarf immer einer Auseinandersetzung mit den strukturellen Bedingungen und dem Problem des „richtigen Verhaltens in den falschen Strukturen“

Eine theoretische Kontextualisierung und Fundierung der Diskurse sowie eine umfassende Analyse der Diskussionen und Konzepte innerhalb der Fachgruppe Ernährungs- und Verbraucherbildung kann an dieser Stelle nicht geleistet werden. Es soll aber darauf hingewiesen werden, dass in den letzten Jahrzehnten fundierte Konzepte mit einer umfassenden (ess-)kulturellen Perspektive für die Ernährungs-, Gesundheits- und Verbraucherbildung an Schulen entstanden sind und auch im Hinblick auf den gesellschaftlichen Wandel ständig weiter entwickelt werden (z.B. Heindl, 2003; Fachgruppe Ernährungs- und Verbraucherbildung, 2005). Der Transfer in die Schulpraxis ist jedoch bisher nicht umfassend gelungen. Im Folgenden wird eine erste Annäherung an die für die Lehrerinnen- und Lehrerbildung relevanten Gesundheitsdiskurse versucht, um daraus hochschuldidaktische Herausforderungen zu formulieren und zu konturieren.

\subsection{Gesundheit als soziales Konstrukt}

Auch wenn „Gesundheit“ eine wichtige Zielgröße naturwissenschaftlicher Forschung ist und viele Gesundheitsfaktoren berechenbar und konkret erklärbar erscheinen (Raacke, 2014, S. 69), so zeigt ein historischer Rückblick deutlich, dass Gesundheit keine bestimmbare und anthropologisch messbare Konstante, sondern eine kollektive zeithistorische Konstruktion darstellt. Die Vorstellung von Gesundheit als einem natur- oder gottgegebenen Gut wandelte sich in modernen Gesellschaften zur Vorstellung von Gesundheit als Ergebnis von individuellem Verhalten; Krankheit wird damit als abweichendes Verhalten verstanden (Briesen, 2010, S. 18; Hahn, 2011, S. 32; Hoefert \& Klotter 2011, S. 7; Raacke, 2014, S. 70). Hahn beschreibt in Anlehnung an Foucault einen Wandel der modernen Gesellschaft, die Gesundheit institutionalisiert und dadurch Normen setzt. So beanspruchen die akademischen Disziplinen der Psychologie, der Medizin und auch der Sozialwissenschaften, die Definitionsmacht darüber, was und wer gesund ist und was als normales oder abweichendes Verhalten gedeutet werden kann. Daraus entwickelt sich eine Rechtfertigungsgrundlage für individuelle und gesellschaftliche Verhaltensregeln, die zunächst noch repressiv umgesetzt wurden, sich später aber zu Gesundheitsnormen weiter entwickelten5 (Hahn, 2011, S. 33; Steindor, 2009, S. 76). 


\section{Lässt sich Gesundheit lehren?}

Mit dem Verständnis des Gesundheitsbegriffs stellt sich also zunächst die Frage nach aktuell geltenden Gesundheitsnormen. Welche sind das, wodurch werden diese bestimmt und legitimiert? Wer soll mit welcher Begründung und mit welchen Mitteln zu einem „besseren, gesünderen“ Verhalten angehalten werden? Wird der Auftrag für eine gesunde Lebensführung vor allem legitimiert durch die Aufrechterhaltung der Arbeitskraft? (Schorb \& Schmidt-Semisch, 2012, S. 53; Schorb, 2013) Programme zur „gesunden Schule" sowie des betrieblichen Gesundheitsmanagements argumentieren beispielsweise zunächst mit Leistungsfähigkeit der Schülerinnen und Schüler bzw. der Angestellten und erst in zweiter Linie mit einem Zugewinn an individueller Lebensqualität (Paulus \& Witteriede, 2008).

Besonders aufschlussreich und interessant ist in diesem Zusammenhang ein Blick auf die sozialen Differenzierungen des Gesundheitsverständnisses. Beispielhaft lässt sich dies am gesellschaftlichen Konzept der „Schlankheit“ nachvollziehen. So gehört es zum verbreiteten Gesundheitsverständnis, Schlankheit mit Gesundheit gleichzusetzen. Hahn und Schorb zeigen jedoch, dass das Dicksein erst ausdrücklich als Gesundheitsproblem wahrgenommen und kommuniziert wurde, als die Armen deutlich zugenommen haben und Übergewicht als ein Problem der Unterschicht identifiziert wurde (Hahn, 2011, S. 41; Schorb, 2010, S. 106). Medizinische Erkenntnisse, dass diese Gleichsetzung zumindest nicht durchgängig haltbar ist, konnten die kollektive Vorstellung bisher nicht erschüttern. Es ist also eine soziale, gesellschaftliche Frage, welche Gesundheitsrisiken thematisiert und problematisiert werden und welche nicht (Schorb \& Schmidt-Semisch, 2012, S. 54)

Da körperbezogene Verhaltensweisen durch soziale Strukturen produziert und reproduziert werden, stellt der Umgang mit dem Körper sowie seine Erscheinungsform auch eine Markierung sozialer Grenzen dar. Das Einhalten von Gesundheitsnormen wird so zum Distinktionsmerkmal, zum sozialen Platzanweiser (Hahn, 2011, S. 43). Bürgerliche Werte wie Disziplin, Mäßigung und Kontrolle kommen mit dem Schlankheitsgebot auch in modernen Gesellschaften durch die Hintertür wieder herein und gesundes, rationales Ernährungsverhalten wird zu einer moralischen Pflicht (Barlösius, 1999, S. 220f.). Schlankheit, Gesundheit und damit auch Lebensglück, werden als Ergebnis von Willenskraft und Disziplin wahrgenommen, gelten damit als herstellbar und als äußeres Zeichen für den Charakter eines Menschen (Zimmer \& Klotter, 2011, S. 5). Steindor beschreibt „Gesundheit“ als ein konstitutives Element bürgerlicher Gesellschaften (Steindor, 2009, 77).

Die Definitionsmacht für das, was gesund und richtig ist, liegt bei den Institutionen des Gesundheitswesens und wird somit durch die gesellschaftliche Mittel- und Oberschicht bestimmt. Den sozialen Unterschichten wird hingegen in allen gesundheitsbezogenen Studien ein ,riskanter Lebensstil“ bescheinigt ${ }^{6}$ (z.B. Robert Koch Institut, 2005). Diese soziale Strukturierung lässt sich auch auf die Institutionen des Bildungswesens übertragen. Lehrerinnen und Lehrer repräsentieren in der Regel eben- 


\section{Lässt sich Gesundheit lehren?}

falls die gesellschaftliche Mittelschicht, wodurch bestimmte Gesundheitsnormen transportiert und in der EVB zu Grunde gelegt werden.

\subsection{Verantwortung für gesunde Lebensführung}

Da Gesundheit in modernen Gesellschaften nicht als persönliches Schicksal, sondern als Folge des eigenen Verhaltens begriffen wird, sind damit auch bestimmte Vorstellungen über die Verantwortung für eine gesunde Lebensführung verbunden. (Hoefert \& Klotter, 2011, S. 7, Raacke, 2014, S. 70, Steindor, 2009, S. 72). Das Individuum wird also für die Herstellung der eigenen Gesundheit verantwortlich gemacht. Persönliche Faktoren, wie zum Beispiel genetische Dispositionen oder Verhältnisfaktoren, die die individuellen Handlungsspielräume begrenzen, stehen als Bedingungsgrößen dahinter zurück (Raacke, 2014, S. 70). Eine Verantwortungszuweisung ohne entsprechende Handlungsspielräume oder soziokulturelle Ressourcen führt allerdings zu Überforderung und Stigmatisierung, wie sich am Beispiel des gesellschaftlichen Umgangs mit Adipositas bzw. den Adipösen zeigt (Schorb, 2012; Methfessel, 2015, S. 93). Die Bedingungen des Aufwachsens in einer modernen Konsumgesellschaft können als adipogen, also als Übergewicht erzeugend, bezeichnet werden (Häußler, 2012, S. 258). Es braucht sowohl materielle und soziokulturelle Ressourcen, als auch bestimmte Wertorientierungen, um innerhalb dieser strukturellen Rahmenbedingungen der gesellschaftlichen Gesundheits- und Schlankheitsnorm zu entsprechen. So investieren besonders Mittelschichtsfamilien (in der Regel die Mütter) viel Zeit und Energie, um ihre Kinder vor den omnipräsenten Verlockungen der Lebensmittelindustrie und vor den medialen Unterhaltungsangeboten zu schützen, um Bewegungsanreize in einem wenig bewegungsförderlichen Alltag in Schule und direkten Wohnumfeld zu schaffen (Leonhäuser, Meier-Gräwe \& Möser et. al. 2009).

Dieser Befund weist auf eine weitere problematische Linie in der Zuschreibung von Gesundheitsverantwortung hin. Schnell stehen die Familien im Blickpunkt der Kritik, da diesen als primärer Sozialisationsinstitution eine wesentliche Bedeutung in der Ernährungs-, Gesundheits- und Verbrauchersozialisation zukommt - „das ist zunächst auch nicht von der Hand zu weisen“ (Sting, 2007, 483). Es ist jedoch Teil des kollektiven Gedankenguts, dass ungesunde Lebens- und Ernährungsweisen oder fehlende Verbraucherkompetenzen ein Ergebnis von Mängeln im Elternhaus sind. Schulen weisen bisher strukturell die Verantwortung von sich und möchten nicht als „Reparaturbetrieb für familiäre Versäumnisse herhalten. Dabei gerät aus dem Blick, dass Familien durch ihre reproduktive Funktion (v. Schweitzer, 1991) bereits enorme Leistungen für das Funktionieren der Gesellschaft erbringen, in dem sie unter anderem die nächste Generation heranziehen und sozialisieren, Kranke pflegen und wieder fit machen für den Arbeitsmarkt. Damit sind sie schon immer ein „Reparaturbetrieb“ für die Gesellschaft und können, auch im eigenen Interesse, bis zu einem gewissen Grad problematische Rahmenbedingungen ausgleichen. Einiges deutet 


\section{Lässt sich Gesundheit lehren?}

jedoch darauf hin, dass die privaten Haushalte diesen Ausgleich nicht mehr umfassend leisten zu können - auch auf gesellschaftlicher Ebene sieht man die Haushaltsarbeit erst, wenn sie nicht gemacht ist („,Krise der Reproduktionsarbeit“ - König \& Jäger, 2011).

Die Ernährungs- und auch Gesundheitsverantwortung in Familien fällt in aller Regel nach wie vor in den Zuständigkeitsbereich von Frauen. Sie werden zum Teil direkt adressiert, fühlen sich aber auch selbst angesprochen, wenn es um gesundheitliche Defizite oder ungünstige Ernährungsweisen geht. Es rührt an der Identität und der Vorstellung von einer „,guten Mutter“, wenn die fürsorglichen Pflichten nicht im Sinne der gesellschaftlichen (Gesundheits-)Normen erfüllt werden können. So lange jedoch die Frage der Vereinbarkeit von Familie und Beruf ebenfalls in den individuellen Verantwortungsbereich von Müttern fällt und als Leistung der eigenen Person verstanden wird, ist dies nur unter großen Anstrengungen und mit entsprechenden Ressourcen zu leisten (Leonhäuser, Meier-Gräwe \& Möser, 2009) Eine Zuschreibung von umfassender (Gesundheits-) Verantwortung an die Familien und damit vor allem an die Mütter ist unter den gegebenen Bedingungen ausgesprochen problematisch und steht in Konflikt mit der Norm der Geschlechtergerechtigkeit.

Die alltägliche Versorgung hat sich verändert. Derzeit findet eine Neujustierung der Grenze zwischen öffentlicher und privater Verantwortung für Versorgungsaufgaben auf der einen Seite und für die Bildungsaufgaben im Bereich der alltäglichen Lebensführung auf der anderen Seite statt, wie es sich zum Beispiel an der Diskussion um Schulverpflegung und Ganztagsschulentwicklung zeigt. Dieser Wandel ist eine zentrale Herausforderung für die Lehrerinnen und- Lehrerbildung im Bereich der EVB, da die Lehrkräfte eine tragende Funktion für die Konzeption und Umsetzung einer geteilten Erziehungs-, Bildungs- und auch Versorgungsverantwortung zwischen den zentralen Sozialisationsinstitutionen Schule und Familien haben.

\section{Fazit: Folgerungen für die Lehramtsausbildung}

Das in den aktuellen Bildungskonzepten definierte Ziel der Entwicklung von Autonomie und Eigenverantwortung ist hochgradig anspruchsvoll. Am Beispiel der Gesundheitsbildung ist deutlich geworden, wie problematisch ein demokratischemanzipatorisches Gesundheitskonzept auf Grundlage von Empowerment ist, wenn Eigenverantwortung und Autonomie nicht erreicht werden kann. Sobald persönliche und soziokulturelle Ressourcen für Bildungsprozesse und letztlich für eine individuelle Verantwortungsübernahme aus dem Blickfeld geraten, wird Überforderung und Stigmatisierung zur anderen Seite der Medaille „Empowerment“.

Durch den Paradigmenwechsel von der Erziehung zur Bildung haben sich die Normativitäten verschoben. So werden in der haushaltsbezogenen Bildung keine konkreten normativen Lerninhalte transportiert, wie zum Beispiel die ,richtige, rationelle Haushaltsführung". Allerdings sind Fragen der alltäglichen Lebensführung immer 


\section{Lässt sich Gesundheit lehren?}

durch normative Zugänge geprägt, die derzeit besonders in Form von bestimmten Gesundheitsvorstellungen und -normen transportiert werden. Diese sind jedoch nicht explizit sichtbar. Für die Lehrerinnen- und Lehrerbildung lassen sich in diesem Kontext vor allem zwei zentrale Aufgaben für die Entwicklung von professioneller Kompetenz in der EVB ableiten:

- Reflektion über Gesundheitsnormen und ihre sozialen Verortung, um die eigenen normativen Überzeugungen zu erkennen und die Bedeutung der eigenen sozialen Herkunft für subjektive Konzepte in der EVB zu verstehen.

- Verständnis über die biografischen Hintergründe von verschiedenen Gesundheits- und Körperkonzepten entwickeln und das Zusammenspiel zwischen individuellem Verhalten und strukturellen Bedingungen verstehen.

Für die Vorbereitung auf die Schulpraxis und die Begleitung wären weitere Erkenntnisse über die „Berufsmoral“ und die „fachbezogenen Beliefs“ (Bender \& Hertrampf, 2014, S. 6) der Lehrkräfte in der EVB sehr hilfreich.

Im Sinne eines konstruktivistischen Bildungsverständnis geht es um die Ausbildung einer kritischen Subjektivität, welche das Verständnis zu Grunde gelegt, dass Lernen immer in Auseinandersetzung mit dem gesellschaftlichen Umfeld stattfindet und Bildung als Möglichkeit verstanden wird, sich in den kulturellen Gegebenheiten zurecht zu finden. Für die Ernährungs-, Gesundheits- und Verbraucherbildung braucht es angemessene Handlungsmodelle, die mit realistischen Erwartungen verknüpft werden. Dabei ist Pluralität bzw. Heterogenität ein wesentliches Merkmal der Sozialisation von Kindern und Jugendlichen, vor allem in alltagsbezogenen Bildungsbereichen.

\section{Anmerkungen}

1 Dies liegt jedoch in der Natur der Sache und ist nicht als Auftrag für Bildungsansätze zu verstehen: Aufgrund der Komplexität alltäglicher Lebensführung und ihren Bedingungen in einer modernen Konsumgesellschaft sind die Ziele und Konzepte von Alltagspraktiken in letzter Instanz nicht objektivierbar; eine durchgängige handlungsrelevante Reflektion von Alltagsroutinen ist lebenspraktisch ebenfalls nicht umsetzbar.

2 Die Umfrage wurde im WS 2014/15 im Rahmen einer Grundlagenvorlesung, die für alle Studierenden des Fachs Alltagskultur und Gesundheit im ersten Semester auf dem Stundenplan steht, an der PH Heidelberg durchgeführt. Sie war als Fragebogen eingebettet in die Veranstaltungsseite innerhalb des elektronischen Campusmanagementportals StudIP. Neben der Frage zur Motivation für die Studienfachwahl wurden unter anderem Fragen zu Interessenfeldern innerhalb der EVB 


\section{Lässt sich Gesundheit lehren?}

und zu schulischen, beruflichen oder privaten Vorerfahrungen gestellt. Es gab sowohl geschlossene wie auch offene Fragen, welche schriftlich beantwortet wurden. Die Teilnahme an der Umfrage war freiwillig, es haben 66 von 170 in der Veranstaltung eingetragenen Studierenden teilgenommen.

3 Dass die Begriffe einer falschen, richtigen oder gesunden Ernährung nicht objektivierbar sind, wird für Studierende in der Regel anhand von konkreten Fragestellungen deutlich, so zum Beispiel in der Diskussion über den Stellenwert von Fleischgerichten in der Nahrungszubereitung. Eine Gruppe wünscht sich mit esskulturell gestützten Argumenten mehr Fleischgerichte, eine andere bringt ökologische und tierethische Argumente für einen rein veganen Nahrungszubereitungsunterricht vor.

4 Die umfassenden bildungstheoretischen Entwicklungslinien und zum Teil konträren Debatten können an dieser Stelle nicht nachgezeichnet werden (z.B. Tenorth, 2008).

5 Als Beispiel beschreibt Hahn, dass zu Zeiten der frühen Industrialisierung Mitte des 19. Jahrhunderts der Umgang der Unterschichten mit ihrem Körper als moralisches Problem und unrationeller Umgang mit Körperkräften formuliert wurde, ohne Bezug auf die prekären, gesundheitsschädlichen Lebens- und Arbeitsbedingungen zu nehmen. Durch staatliche Überwachung der Gesundheitspflege wurde ein Hygieneverhalten erreicht, was zu einer Eindämmung von Epidemien wie Cholera, Typhus und Pocken führte (Hahn, 2011, S. 33).

6 Niewöhner interpretiert das T-Shirt-Statement „Bier formte diesen wunderschönen Körper" als Widerstand gegen das mächtige Körperkonzept der Schlankheit in modernen Gesellschaften und damit als Ausdruck sozialer Abgrenzung (Hahn, 2011, S. 43; Niewöhner, 2008, S. 113 ).

\section{Literatur}

Barlösius, E. (1999). Soziologie des Essens. Eine sozial- und kulturwissenschaftliche Einführung in die Ernährungsforschung. Weinheim und München: Juventa.

Bartsch, S. (2012). Subjektorientierung. Ein Beitrag zur kompetenzorientierten Aufgabengestaltung in der Verbraucherbildung. Haushalt in Bildung \& Forschung, 1(3), 52-64.

Bartsch, S., Büning-Fesel, M \& Cremer, M. (2013): Ernährungsbildung - Standort und Perspektiven. Ernährungs Umschau, 61(2), M84-95.

Bender, U. \& Hertrampf, A. (2014): Fachbezogene moralische Überzeugungen von Lehrpersonen in der Ernährungs- und Verbraucherbildung (EVB). Haushalt in Bildung \& Forschung, 3(4), 3-15.

Briesen, D. (2010). Das gesunde Leben. Ernährung und Gesundheit seit dem 18. Jahrhundert. Frankfurt am Main: Campus. 


\section{Lässt sich Gesundheit lehren? |}

Fachgruppe Ernährung und Verbraucherbildung (2005). Schlussbericht REVIS Modellprojekt. Reform der Ernährungs- und Verbraucherbildung an Schulen. [http://dsg.uni-paderborn.de/evb/forschung-und-entwicklung/revisstartseite/revis-schlussbericht/]

Hahn, D. (2010). Prinzip Selbstverantwortung? Eine Gesundheit für alle? Jahrbuch für Kritische Medizin und Gesundheitswissenschaften: VerantwortungSchuld - Sühne, 46. 29-50.

Häußler, A. (2012). Verhältnisprävention in einer adipogenen Umwelt. Ernährungs Umschau, 60(5), 258-263

Heindl, I. (2003). Studienbuch Ernährungsbildung. Ein europäisches Konzept zur schulischen Gesundheitsförderung. Bad Heilbrunn: Julius Klinkhardt.

Hoefert, H.-W. \& Klotter, C. (2011). Gesunde Lebensführung - kritische Analyse eines populären Konzepts. Bern, Göttingen: Huber.

Klafki, W. (2007). Neue Studien zur Bildungstheorie und Didaktik. Zeitgemäße Allgemeinbildung und kritisch-konstruktive Didaktik (6. Aufl.). Weinheim und Basel: Beltz.

König, T. \& Jäger, U. (2011). Reproduktionsarbeit in der Krise und neue Momente der Geschlechterordnung: Alle nach ihren Fähigkeiten und ihren Bedürfnissen!. In A. Demirovic, J. Dück, F. Becker, \& P. Bader (Hrsg.), VielfachKrise. Im finanzdominierten Kapitalismus. (In Kooperation mit dem Wissenschaftichen Beirat von Attac) (S. 147-164). Hamburg: VSA.

Leonhäuser, I.-U., Meier-Gräwe, U. \& Möser, A. et al. (2009). Essalltag in Familien. Ernährungsversorgung zwischen privatem und öffentlichen Raum. Wiesbaden: VS-Verlag.

Methfessel, B. (2015). Welche Moral hätten Sie denn gerne? Essen im Konflikt zwischen den unterschiedlichen Anforderungen an die Lebensführung. In G. Hirschfelder, A. Ploeger, J. Rückert-John et al. (Hrsg.), Was der Mensch essen darf. Ökonomischer Zwang, ökologisches Gewissen und globale Konflikte (S. 83-100). Wiesbaden: VS-Verlag.

Paulus, P. \& Witteriede, H. (2008): Schule-Gesundheit-Bildung: Bilanz und Perspektiven. Projektbericht.

[www.baua.de/de/Publikationen/Fachbeitraege/F2033.pdf].

Raacke, G. (2014). Wer früher stirbt, ist selber schuld! Ein kritischer Blick auf das aktuelle Gesundheitsregime. Haushalt in Bildung \& Forschung, 3(4), 69-79.

Robert Koch Institut (RKI) (Hrsg.) (2005). Armut, soziale Ungleichheit und Gesundheit. Expertise des Robert Koch-Instituts zum 2. Armuts- und Reichtumsbericht der Bundesregierung. Beiträge zur Gesundheitsberichterstattung des Bundes. Berlin.

Schlegel-Matthies, K. (2004). Verbraucherbildung im Forschungsprojekt REVIS Grundlagen. Paderborner Schriften zur Ernährungs- und Verbraucherbildung. 


\section{Lässt sich Gesundheit lehren?}

Schorb, F. (2013). IN FORM - Nachhaltig fit und schlank für den Standort Deutschland? Gesundheit Berlin Brandenburg (Hrsg.). Dokumentation 18. Kongress Armut und Gesundheit. Brücken bauen zwischen Wissen und Handeln - Strategien der Gesundheitsförderung. Berlin.

Schorb, F. \& Schmidt-Semisch, H. (2012). Die Problematisierung gesundheitlicher Risiken. In A. Hanses \& K. Sander (Hrsg.), Interaktionsordnungen. Gesundheit als soziale Praxis (S. 53-69). Wiesbaden: VS-Verlag.

Schorb, F. (2010). Fit for fun? Schlankheit als Sozialprestige. In B. Paul \& H.

Schmidt-Semisch (Hrsg.), Risiko Gesundheit. Über Risiken und Nebenwirkungen der Gesundheitsgesellschaft (S. 105-121). Wiesbaden: VS-Verlag.

Steindor, M. (2009). Gerechte Gesundheitschancen. Von der Entwicklung eines gesundheitsfördernden Lebensstils für die Bürger bis hin zur Gesundheitspädagogik für die Arbeiterschicht. In U. Bittlingmeyer, D. Sahrai \& P.-E. Schnabel (Hrsg.). Normativität und Public Health: vergessene Dimensionen sozialer Ungleichheit (S. 75-109). VS-Verlag, Wiesbaden.

Sting, S. (2007). Gesundheit. In J. Ecarius (Hrsg.) Handbuch Familie (S. 480-499). Wiesbaden: VS-Verlag.

Suter, C. \& Högger, D. (2014). Ernährungsbildung - was sollen, dürfen und müssen Lehrerinnen und Lehrer? Haushalt in Bildung \& Forschung, 3(4), 16-27.

Tenorth, H.-E. (2008). Bildungstheorie angesichts von Basiskompetenzen. Über die Ignoranz des Selbstverständlichen im Prozess des Aufwachsens. Zeitschrift für pädagogische Historiographie, 14(1), 26-31.

Tenorth, H.-E. \& Tippelt, R. (Hrsg.). (2007). Bildung, Beltz Lexikon Pädagogik (S. 92-95). Weinheim und Basel: Beltz-Verlag.

v. Schweitzer, R. (1991). Einführung in die Wirtschaftslehre des privaten Haushalts. Stuttgart: UTB

Zimmer, S. \& Klotter, C. (2011). Die protestantische Ethik als „Geist“ der Gesundheitsförderung? IAKE Mitteilungen (18), 2-10.

\section{Verfasserin}

Prof. ${ }^{\text {in }}$ Dr. Angela Häußler

Pädagogische Hochschule Heidelberg

Alltagskultur und Gesundheit

Im Neuenheimer Feld 561

D-69120 Heidelberg

E-Mail: a.haeussler@ph-heidelberg.de

Internet: www.ph-heidelberg.de/alltagskultur-und-gesundheit/alltagskultur-undgesundheit.html 